\title{
UBVI CCD PHOTOMETRY OF THE OPEN CLUSTERS NGC 4609 AND HOGG 15
}

\author{
Seung-Hwa Kook ${ }^{1}$, Hwankyung Sung ${ }^{1}$, And M. S. Bessell ${ }^{2}$ \\ ${ }^{1}$ Department of Astronomy and Space Science, Sejong University, 98 Kunja-dong, Kwangjin-gu, Seoul 143-747, \\ Korea \\ E-mail: sungh@sejong.ac.kr \\ 2 Research School of Astronomy and Astrophysics, Australian National University, MSO, Cotter Road, Weston, \\ ACT 2611, Australia \\ E-mail: bessell@mso.anu.edu.au \\ (Received July 12, 2010; Revised August 18, 2010; Accepted August 25, 2010)
}

\begin{abstract}
$U B V I$ CCD photometry is obtained for the open clusters NGC 4609 and Hogg 15 in Crux. For NGC 4609, CCD data are presented for the first time. From new photometry we derive the reddening, distance modulus and age of each cluster - NGC $4609: E(B-V)=0.37 \pm 0.03, V_{0}-M_{V}=10.60 \pm$ $0.08, \log \tau=7.7 \pm 0.1 ; \operatorname{Hogg} 15: E(B-V)=1.13 \pm 0.11, V_{0}-M_{V}=12.50 \pm 0.15, \log \tau \lesssim 6.6$. The young age of Hogg 15 strongly implies that WR 47 is a member of the cluster. We also determine the mass function of these clusters and obtain a slope $\Gamma=-1.2( \pm 0.3)$ for NGC 4609 which is normal and a somewhat shallow slope $(\Gamma=-0.95 \pm 0.5)$ for Hogg 15.
\end{abstract}

Key words : color-magnitude diagrams (H-R diagram) — open clusters and associations: individual (NGC 4609 \& Hogg 15)

\section{INTRODUCTION}

Although massive stars play an important role in the chemical and dynamical evolution of a galaxy, knowledge relating to their formation processes and environments is still very scanty due to the rareness of massive O, Wolf-Rayet (WR), and luminous blue variable (LBV) stars. From the correlation between WR stars and the turn-off mass of young open clusters in the Galaxy, Massey et al. (2001) found that early-type WN (WNE) stars appear to have evolved from a large range of high mass stars, whereas WN7 or LBVs are only found in clusters with the highest turn-off masses.

WR 47 (= HDE $311884-\mathrm{WN} 6+\mathrm{O} 5: \mathrm{V})$ is located at the periphery of the compact open cluster Hogg 15. The relationship between WR 47 and Hogg 15 is a key to studying the evolutionary status and progenitor mass of WR 47. The double-line binary system WR 47 has attracted many observations because the system consists of very massive stars with a relatively high mass ratio $\left(q_{W R} \equiv \frac{m_{\mathrm{WR}}}{m_{\mathrm{O}}+m_{\mathrm{WR}}}=0.85\right)$ (van der Hucht 2001). Niemela et al. (1980) found that the WN6 star in WR 47 is more massive than $40 \mathrm{M}_{\odot}$ from the orbit of the double-line binary system. Lamontagne \& Moffat (1987) detected about 0.1 mag variability for WR 47, and showed that the variability was not caused by the pulsation-like intrinsic variability of WR stars. Later, Moffat et al. (1990) determined the mass of

Corresponding Author: H. Sung each component and the mass loss rate from the revised ephemeris based on photometry and polarimetry. The masses they determined were $48 \pm 5 \mathrm{M}_{\odot}$ for WN6 and $57 \pm 6 \mathrm{M}_{\odot}$ for $\mathrm{O} 5: \mathrm{V}$. Lamontagne et al. (1996) revised the mass of each component $\left(51 \mathrm{M}_{\odot}\right.$ for $\mathrm{WN} 6$ and $60 \mathrm{M}_{\odot}$ for $\left.\mathrm{O} 5: \mathrm{V}\right)$. The mass of the companion is somewhat larger than its spectroscopic or evolutionary mass $\left(\sim 40 \mathrm{M}_{\odot}\right)$. The mass of each component is strongly dependent on the geometry of the system, in particular, the adopted inclination angle.

Feinstein \& Marraco (1971) determined the distance $(d=1320 \mathrm{pc})$ and age $\left(6 \times 10^{7} \mathrm{yrs}\right)$ of NGC 4609 , and found a somewhat large spread around its main sequence. Muzzio et al. (1976) and Lodén (1979) studied this region as part of a project studying the loose clustering of young stars or the spiral arm structure of the Galaxy. Because NGC 4609 is an intermediateage open cluster at a relatively large distance with few members, it did not attract modern CCD observation until now. Moffat (1974) performed $U B V$ photoelectric photometry of 23 stars in Hogg 15, and determined the distance $(d=4.2 \pm 0.4 \mathrm{kpc})$ and age $\left(\lesssim 8 \times 10^{6} \mathrm{yrs}\right)$ of the cluster. He concluded that the WN6 star HDE 311884 is likely to be a member of Hogg 15 .

Orsatti et al. (1998) performed polarimetry on 23 stars in Hogg 15. They established the existence of intracluster dust associated with Hogg 15 with a slightly different grain size distribution. They also showed WR 47 to be highly polarized with a strong time variation. Sagar et al. (2001) and Piatti \& Clariá (2001) per- 
formed CCD photometry for the compact open cluster Hogg 15. Interestingly they obtained very discrepant results. Sagar et al. (2001) derived the distance and age of Hogg 15 as $3.0 \pm 0.3 \mathrm{kpc}$ and $6 \pm 2 \mathrm{Myr}$, respectively. While Piatti \& Clariá (2001) determined $2.6 \pm$ $0.08 \mathrm{kpc}$ and $\sim 300 \mathrm{Myr}$, respectively. From their relatively large age for Hogg 15, Piatti \& Clariá (2001) ruled out WR 47 being a cluster member. One aim of our work is to disentangle the discrepancy between the results of Sagar et al. (2001) and Piatti \& Clariá (2001).

In Section 2 we present the $U B V I$ photometric data for 7208 stars. We compare our data with those of previous studies. The reddening law of the observed region is derived in Section 3. From the surface density variation of candidate cluster stars we determine the radius of NGC 4609 and Hogg 15. The photometric members for both clusters are selected in the same section. The age and mass function are derived in Section 4 . The discussion and summary are presented in Section 5 and Section 6 respectively.

\section{OBSERVATIONS AND DATA REDUC- TION}

$U B V I$ CCD photometry for NGC 4609 and the Hogg 15 region was performed during two nights in 1997 March using the 1-m telescope at Siding Spring Observatory. On March 2, due to limited observing time and the night not being photometric, we could observe the region only in $U B V$. We therefore observed the region again on March 4 in $U B V I$. We obtained images for two sets of exposure times: long, $2 \times 30 \mathrm{~s}$ in $I, 2 \times 60 s$ in $V, 2 \times 100 s$ in $B$, and $2 \times 300 s$ in $U$; and short, $5 s$ in $V$ and $I, 10 s$ in $B$, and $30 s$ in $U$. We also observed two Landolt standard regions (SA 98 \& PG 1312-086) on March 4. The standard transformation relations in Sung, Bessell \& Lee (1998) are used for $U B V$, but for the $I$ transformation we used the relation in Sung et al. (2008). The atmospheric extinction coefficients on March 4 were 0.094 ( \pm 0.011$), 0.190( \pm$ $0.013), 0.355( \pm 0.008)$, and $0.580( \pm 0.019)$ in $I, V$, $B$, and $U$, respectively. The secondary extinction coefficients in $B$ and $U$ from Sung et al. (1998) have been adopted and used.

Instrumental magnitudes were obtained using IRAF / DAOPHOT using point spread function fitting. All the instrumental magnitudes were transformed to the standard $U B V I$ system using Landolt (1992) standard stars observed on 1997 March 4 . The photometric data for 7208 stars were obtained and a sample of the data is presented in Table 1. Fig. 1 is the finding chart for stars brighter than $V=17 \mathrm{mag}$. The only saturated star was the peculiar Be star HD 110432, which shows an enormously high X-ray temperature (see Smith \& Balona 2006). The data for HD 110432 were obtained from the SIMBAD database. We have also identified 2MASS counterparts of the optical sources. The star ID 5599 is very red in the optical as well as in the near-

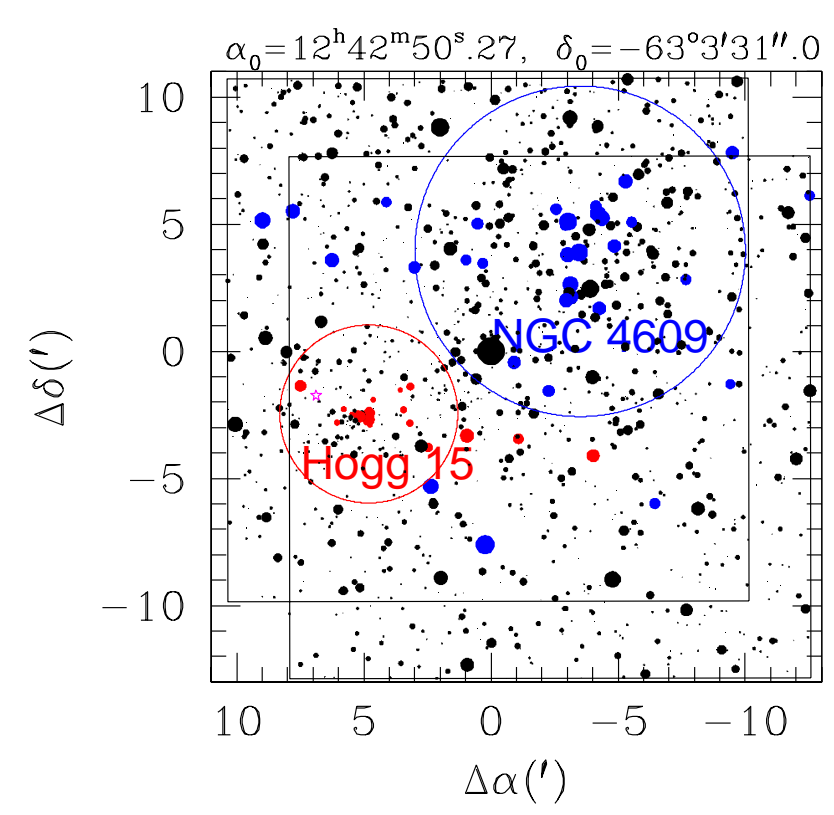

Fig. 1. - Finding chart, centered at HD $110432\left(\alpha=12^{h}\right.$ $42^{m} 20 .^{s} 3, \delta=-63^{\circ} 3^{\prime} 31^{\prime \prime} 0$, J2000.0). The size of the symbol is proportional to the magnitude of the star. Blue and red-colored symbols represent less reddened nearby $(E(B-V)=0.3-0.45 \& d \approx 1.3 \mathrm{kpc})$ and more highly reddened distant $(E(B-V)=1.0-1.5 \mathrm{mag} \& d \approx 3.2$ $\mathrm{kpc}$ ) stars, respectively. The circles represent the radius of NGC 4609 and Hogg 15, respectively. Two large squares show the field of view (FOV) of the two observations. The square slightly shifted to the northeast is the FOV of the 1997 March 2 observation ( $U B V$ only) and that slightly shifted to the southwest is the FOV of the 1997 March 4 observation $(U B V I)$.

IR 2MASS $J H K_{s}$. A spectrum of the star obtained with WiFeS on the $2.3 \mathrm{~m}$ telescope at SSO (see Fig. 2) on 2010 July 19 shows strong TiO and VO bands. The star is clearly a Mira.

The $U B V I$ magnitudes and colors from our study are compared with the previous photoelectric or CCD photometry of Feinstein \& Marraco (1971), Moffat (1974), Muzzio et al. (1976), Lodén (1979), Piatti \& Clariá (1981), and Sagar et al. (2001) in Table 2. The meaning of $\Delta$ is in the sense of our data minus that of the others. Piatti \& Clariá (2001) observed many standard regions from Landolt (1992), but the others, except Moffat (1974)*, used SAAO E-regions as standards. Although our system is tightly matched to the Landolt system, the difference between our data and others is very small in $B-V$. The difference in $U-B$ should be more negative as the $U-B$ scale of the Landolt system is bluer for blue stars (Menzies et al. 1991; Lim et al. 2009). The data by Sagar et al. (2001) do

* No information on the standard stars they observed is available. 
Table 1.

Photometric data ${ }^{\mathrm{a}}$

\begin{tabular}{|c|c|c|c|c|c|c|c|c|c|c|c|c|c|}
\hline$\alpha(J 2000.0)$ & $\delta(J 2000.0)$ & $V$ & $V-I$ & $B-V$ & $U-B$ & $\epsilon_{V}$ & $\epsilon_{V-I}$ & $\epsilon_{B-V}$ & $\epsilon_{U-B}$ & $\mathrm{n}_{o b s}$ & 2MASS & Sp type & Remark \\
\hline $1^{\mathrm{b}} 12: 42: 50.27$ & $-63: 03: 31.0$ & 5.316 & & 0.248 & -0.813 & 0.052 & & 0.018 & 0.027 & 7076 & $12425028-6303310$ & $\mathrm{~B} 2 \mathrm{p}$ & HD 110432 \\
\hline $3902 \quad 12: 42: 52.28$ & $-63: 11: 07.3$ & 9.024 & 0.410 & 0.239 & -0.348 & 0.002 & 0.005 & 0.004 & 0.004 & $\begin{array}{llll}1 & 1 & 1 & 1\end{array}$ & $12425228-6311072$ & B2.5IV-V & HD 110433 \\
\hline 4573 12:43:07.85 & $-62: 54: 42.1$ & 9.347 & - & 1.455 & 1.476 & 0.002 & - & 0.006 & 0.006 & $\begin{array}{llll}1 & 0 & 1 & 2\end{array}$ & $12430790-6254425$ & & HD 110478 \\
\hline 2331 12:42:15.81 & $-63: 01: 03.1$ & 9.555 & 0.768 & 0.709 & 0.362 & 0.002 & 0.006 & 0.004 & 0.004 & $\begin{array}{lllll}2 & 1 & 1 & 1\end{array}$ & $12421586-6301035$ & & \\
\hline 803 11:02:48.36 & $-61: 13: 51.6$ & 9.812 & 0.128 & 0.098 & -0.507 & 0.014 & 0.027 & 0.016 & 0.014 & 11111 & $11024831-6113512$ & & \\
\hline $2668 \quad 12: 42: 23.60$ & $-62: 58: 24.7$ & 9.577 & 0.280 & 0.149 & -0.438 & 0.004 & 0.005 & 0.005 & 0.003 & 2211 & $12422361-6258249$ & Do & 10373 \\
\hline $2506 \quad 12: 42: 19.74$ & $-62: 59: 37.5$ & 9.605 & 0.308 & 0.188 & -0.359 & 0.003 & 0.003 & 0.004 & 0.004 & $\begin{array}{llll}1 & 1 & 1 & 1\end{array}$ & $12421975-6259376$ & & \\
\hline 6544 12:43:51.03 & $-63: 05: 14.9$ & 10.757 & 1.437 & 0.837 & -0.190 & 0.004 & 0.005 & 0.004 & 0.015 & 2222 & $12435102-6305148$ & $05 \mathrm{~V}$ & HDE: \\
\hline 5599 12:43:29.91 & $-62: 57: 36.7$ & 14.224 & 5.803 & 2.177 & 0.568 & 0.004 & 0.008 & 0.011 & 0.013 & 3132 & $12432995-6257369$ & Mira & \\
\hline
\end{tabular}

a This is a sample of the full table, which is available from HS.

${ }^{\mathrm{b}}$ Photometric data from the SIMBAD database (http://simbad.u-strasbg.fr/simbad/)

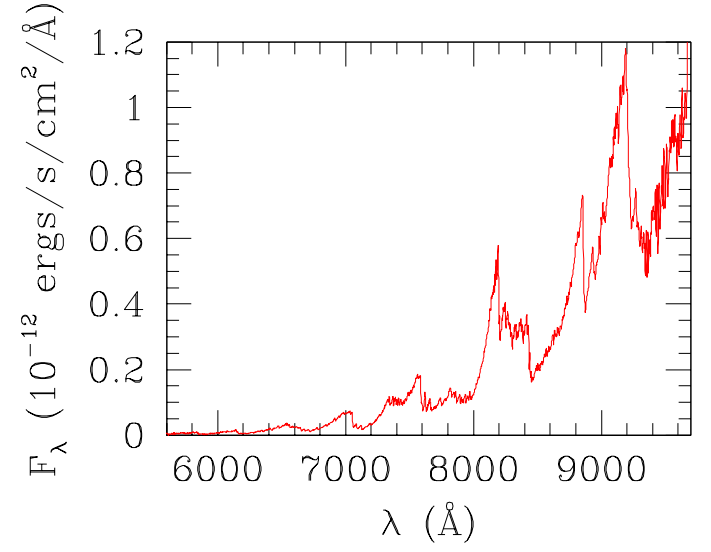

Fig. 2.- Spectrum of ID 5599 obtained with WiFeS on the $2.3 \mathrm{~m}$ telescope at Siding Spring Observatory.

not show such a trend although they observed SAAO Eregions as standard stars. The difference in $V$ or $V-I$ of Piatti \& Clariá (2001) is very large and systematic (see Fig. 3). The source of such a systematic difference cannot be traced back because they did not provide the atmospheric extinction coefficients and transformation coefficients. The difference in $B-V$ is, on the other hand, not as systematic (see Table 2). The $V$ and $V-I$ scales of Sagar et al. (2001) do not show any systematic differences, but do show somewhat large shifts in zero points.

\section{PHOTOMETRIC DIAGRAMS}

\subsection{Reddening Law}

Correction for interstellar reddening is the first step in determining the distance and age of open clusters. Fig. 4 shows the color-color diagrams of the observed region. Evidently there are at least two groups of reddened blue stars. The less reddened blue stars are the
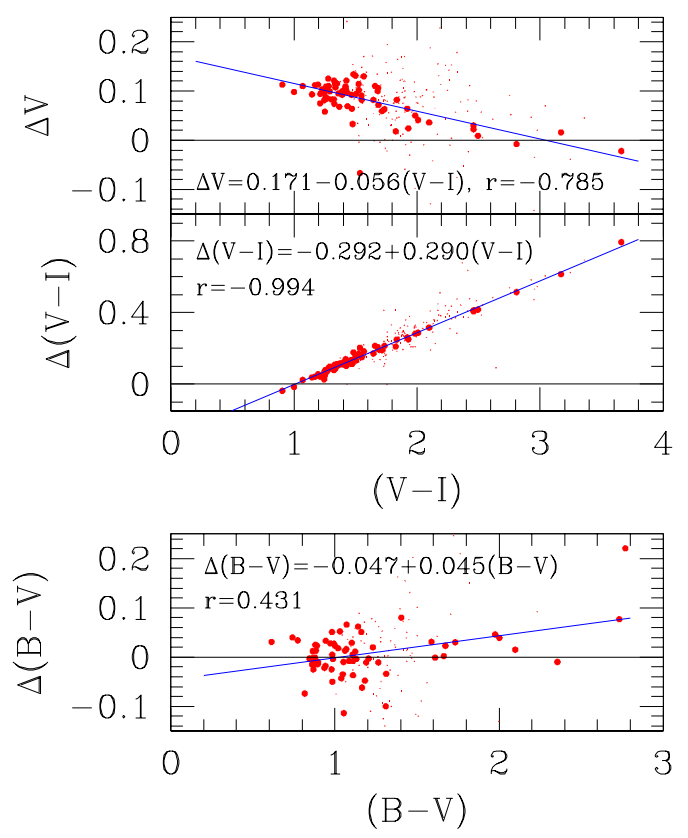

Fig. 3.- Comparison of our photometry with that of Piatti \& Clariá (2001). Large and small dots represent bright ( $V \leq 17 \mathrm{mag})$ and faint stars $(V>17 \mathrm{mag})$, respectively. The regressions were performed only for bright stars. There are large systematic differences in $V$ and $V-I$ between our data and those of Piatti \& Clariá (2001), but the difference in $B-V$ is not as evident.

B type stars belonging to or around the intermediateage open cluster NGC 4609, while the more highly reddened group of stars are stars in and around the young open cluster Hogg 15. Most blue stars in NGC 4609 show little scatter in reddening, but those belonging to Hogg 15 show a large scatter. Unlike WR 37 in the Ruprecht 93 region (Cheon et al. 2010), the $U B V$ colors of WR 47 is very similar to those of normal early 
Table 2.

Comparison with other photometry

\begin{tabular}{|c|c|c|c|c|c|c|c|c|}
\hline Author & $\Delta V$ & $\mathrm{n}\left(\mathrm{n}_{\mathrm{ex}}\right)^{\mathrm{a}}$ & $\Delta(B-V)$ & $\mathrm{n}\left(\mathrm{n}_{\mathrm{ex}}\right)^{\mathrm{a}}$ & $\Delta(U-B)$ & $\mathrm{n}\left(\mathrm{n}_{\mathrm{ex}}\right)^{\mathrm{a}}$ & $\Delta(V-I)$ & $\mathrm{n}\left(\mathrm{n}_{\mathrm{ex}}\right)^{\mathrm{a}}$ \\
\hline Feinstein \& Marraco (1971) & $+0.032 \pm 0.047$ & $58(8)$ & $-0.001 \pm 0.027$ & $60(6)$ & $-0.046 \pm 0.058$ & $57(8)$ & . & \\
\hline Moffat (1974) & $+0.123 \pm 0.225$ & $22(1)$ & $-0.007 \pm 0.063$ & $21(2)$ & $-0.005 \pm 0.126$ & $20(3)$ & $\cdots$ & \\
\hline Muzzio et al.(1976) & $+0.026 \pm 0.122$ & $6(1)$ & $+0.009 \pm 0.017$ & $6(1)$ & $-0.081 \pm 0.041$ & $6(1)$ & ... & \\
\hline Lodén (1979) & $-0.008 \pm 0.019$ & $17(0)$ & $+0.003 \pm 0.014$ & $17(0)$ & $-0.027 \pm 0.028$ & $16(1)$ & $\cdots$ & \\
\hline Piatti \& Clariá $(2001)^{b}$ & see Fig. 3 & $67(1)$ & $+0.006 \pm 0.046$ & $67(1)$ & .. & & see Fig. 3 & $67(1)$ \\
\hline Sagar et al. $(2001)^{\mathrm{b}}$ & $+0.105 \pm 0.022$ & $46(8)$ & $-0.034 \pm 0.023$ & $47(4)$ & $-0.006 \pm 0.063$ & $37(2)$ & $+0.066 \pm 0.027$ & $51(3)$ \\
\hline
\end{tabular}

a The number of stars excluded in the comparison.

${ }^{\mathrm{b}}$ Comparisons were made only for $V \leq 17 \mathrm{mag}$.
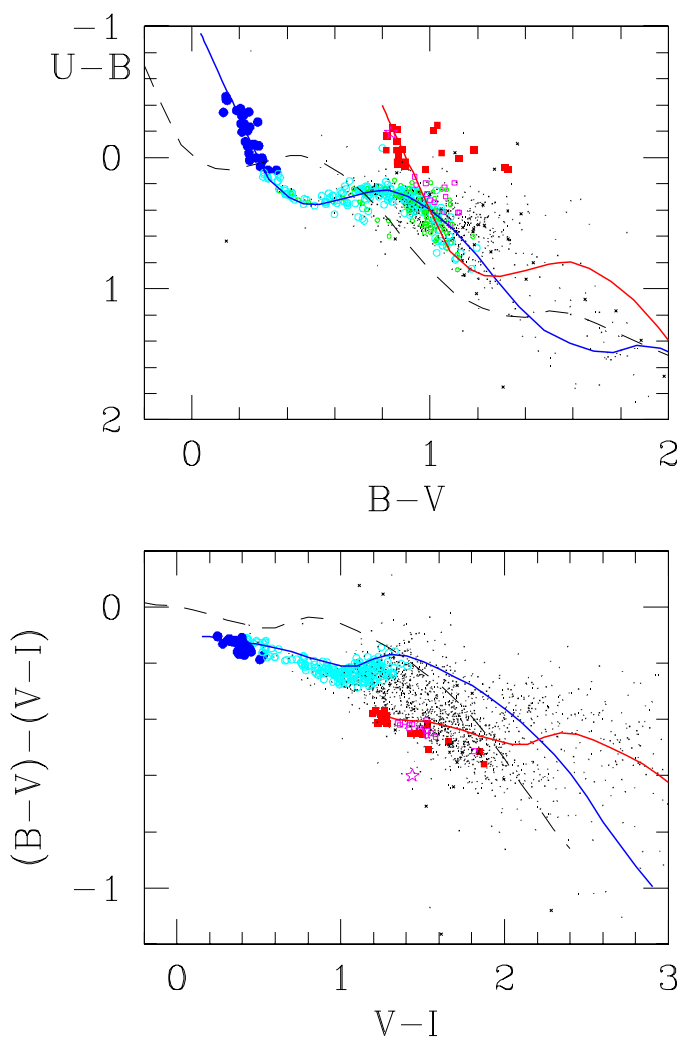

Fig. 4. - The $(U-B, B-V)$ and $(B-V, V-I)$ color-color diagrams. The thin dashed line represents the ZAMS relation, while the two solid lines represent the ZAMS relations reddened by $E(B-V)=0.37$ (blue) and 1.13 (red), respectively. (Blue) Filled circles and (red) squares are the stars belonging to these two groups, while open symbols are the photometric members of each group (see Section 3.3 for the selection criteria of photometric members). The smallest (green) circle represents the photometric members selected only from the $(V, B-V)$ diagram. The star symbol (magenta) denotes WR 47. Small dots and crosses represent, respectively, good data $(\epsilon<0.1)$ and bad data $(\epsilon \geq 0.1)$. type stars in Hogg 15 . The mean $E(B-V)$ of NGC 4609 and Hogg 15 is $0.37( \pm 0.03) \mathrm{mag}$ and $1.17( \pm$ $0.11)$ mag, respectively. The median $E(B-V)$ of Hogg 15 is 1.13 mag. The spatial distribution of the highly reddened group in Fig. 1 is not circular but elliptical in shape.

There are three reddened blue stars between the two reddened zero-age main sequence (ZAMS) relations. The one on the upper left part of the $(U-B$, $B-V)$ diagram is HD 110432 , the brightest star in the observed region. Although the star is in front of NGC $4609\left(\pi_{\text {HD }} 110432=3.32 \pm 0.56\right.$ mas $)$, the reddening $E(B-V)$ is $0.58 \mathrm{mag}$. This means that more than $0.28 \mathrm{mag}$ in $E(B-V)$ (i.e. $E(B-V)$ HD 110432 - $\left.E(B-V)_{\mathrm{NGC} 4609}^{\min }=0.58-0.30=0.28\right)$ is caused by the circumstellar material around HD 110432. The other two stars between the two reddened ZAMS lines are ID 3521 and ID 3585. The distance modulus from ZAMS fitting is between $11.0 \mathrm{mag}$ and $11.7 \mathrm{mag}$ which is very similar to the distance modulus of the Cen OB1 association (Humphreys 1978). A few stars in the observed region may therefore be young stars belonging to the Cen OB 1 association.

Knowledge about the reddening law, especially the total-to-selective extinction ratio, $R_{V} \equiv A_{V} / E(B-$ $V)$, is very important in estimating the distance to an object. We have employed the same method as used in the Ruprecht 93 region (Cheon et al. 2010). The reddening $E(B-V)$ of individual early type stars is calculated from the color-color diagram in Fig. 4. The color excess in each color is calculated using the relation between intrinsic colors.

Fig. 5 shows the relation between $E(B-V)$ and color excesses in other colors. The thick solid line represents the color excess relation for the normal case, i.e. $R_{V}=3.1$. Evidently early type stars in the observed region are slightly elevated above the normal values. The median value of $E(V-I) / E(B-V)$, $E(V-J) / E(B-V), E(V-H) / E(B-V)$, and $E\left(V-K_{s}\right) / E(B-V)$ for $E(B-V) \geq 1.3 \mathrm{mag}$ is $1.360 \pm$ $0.024,2.363 \pm 0.067,2.692 \pm 0.066$, and $2.882 \pm 0.054$, respectively. If we adopt the relation between the color excess ratio and $R_{V}$ of Guetter \& Vrba (1989), the 

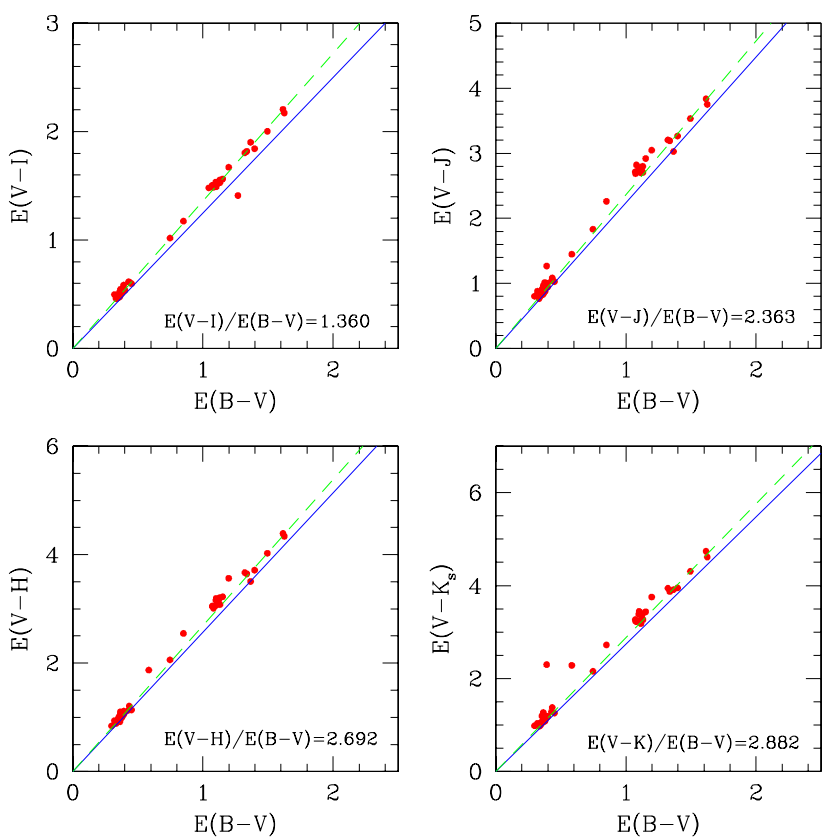

Fig. 5.- Color excess ratios. The color excess of each color is calculated using the relation between intrinsic colors. The thick solid line represents the color excess relation for the normal reddening law, i.e. $R_{V}=3.1$, while the dashed line denotes the color excess ratio for the observed region $\left(R_{V}=3.2\right)$.

color excess ratios give $R_{V}=3.18 \pm 0.06$. We adopt $R_{V}=3.2$ for the observed region. Several blue stars in Hogg $15(E(B-V) \approx 1.1 \mathrm{mag})$ show slightly higher values in $E(V-J), E(V-H)$, and $E\left(V-K_{s}\right)$, but normal values in $E(V-I)$. This may be caused by scattered light or the lower angular resolution of the telescope used in 2MASS. But we cannot rule out the possibility that the $R_{V}$ at the center of Hogg 15 is slightly larger than normal (see discussion in Section 3.3.3 or Orsati et al. 1998).

\subsection{Surface Density Variation and Radius}

As there is a spiral arm at about $1 \mathrm{kpc}$ in the direction of NGC 4609 (see Fig. 5 of Hou et al. 2009) and the distance to NGC 4609 is about $1.3 \mathrm{kpc}$, it is reasonable to expect contamination from spiral arm field stars in the color-magnitude diagram (CMD) of NGC 4609. Since there is no proper motion or other membership study for NGC 4609 and Hogg 15, surface density variation is the only way to determine the radius of each cluster (Sung et al. 1999). ${ }^{\dagger}$

${ }^{\dagger}$ We have checked proper motion data in the Tycho catalogue, but could not find any concentration of candidate cluster stars in the $\left(\mu_{\alpha}, \mu_{\delta}\right)$ plane probably because the proper motion of cluster stars is similar to that of field stars in the Sgr-Car arm.
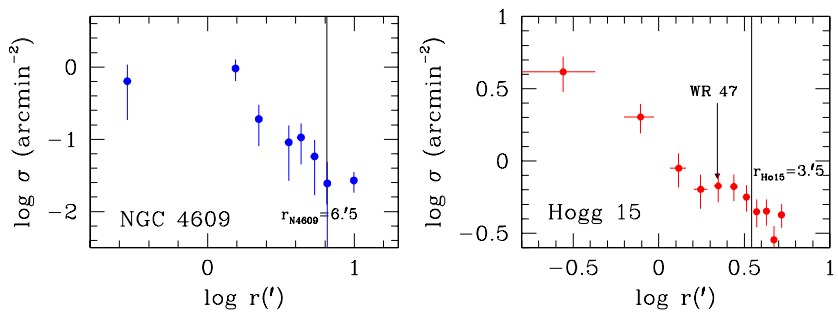

Fig. 6. - The surface density variation of blue stars in NGC 4609 (left) and of stars near the compact young cluster Hogg 15 (right).

For NGC 4609 we have calculated the surface density of blue stars in annuli from the apparent center of NGC $4609\left(\alpha=12^{h} 42^{m} 19 .^{s} 4, \delta=-62^{\circ} 59^{\prime} 35^{\prime \prime}\right)$. The left panel of Fig. 6 shows the radial variation of the surface density of blue stars. The surface density of blue stars decreases rapidly and reaches the surface density of the field at $r \approx 6 .^{\prime} 5$. We tentatively adopt the radius of NGC 4609 as $6 .^{\prime} 5$.

Hogg 15 is a small compact open cluster and the number of early type members is therefore very small. To determine the radius of Hogg 15, we have calculated the surface density of stars with $V=10-17$ mag and $B-V=0.7-1.4 \mathrm{mag}$ from the centroid of early type member stars (red dots in Fig. 1). We have adopted the center of Hogg 15 to be $\alpha=12^{h} 43^{m} 32 .^{s} 8, \delta=$ $-63^{\circ} 5^{\prime} 59^{\prime \prime}$. The variation of surface density is shown in the right panel of Fig. 6 . The surface density decreases rapidly up to $r \approx 1 .^{\prime} 7$, shows a plateau between $r=$ $1 .^{\prime} 7-3 .^{\prime} 0$, and decreases to the background level at $r$ $=3 .^{\prime} 5$. WR 47 is about $2 .^{\prime} 2$ from the adopted center. From surface density variations, Hogg 15 is a cluster with a core and a halo surrounding the core. WR 47 is in the halo of Hogg 15.

\subsection{Photometric Members and Distance}

\subsubsection{Membership Selection Criteria}

Sung \& Bessell (1999) have devised photometric membership selection criteria. The method makes use of a merit of multicolor photometry - the different ZAMS relations for different colors and the different effects of reddening on different colors. The method involves first calculating the distance moduli both in the $\left[V_{0},(B-V)_{0}\right]$ and $\left[V_{0},(V-I)_{0}\right]$ diagrams after correcting for the reddening of the star. Then, one calculates the average and differences in the distance moduli for a given star. Ideally, members of a cluster should have the same distance moduli. In actual fact, due to photometric errors the distance moduli differ slightly. In addition, metallicity (in the case of M35 - Sung \& Bessell 1999) or chromospheric activity (in the case of NGC 2516 - Sung et al. 2002) also affects the differences in distance moduli because they have 
different effects on different colors. We select the members of a cluster if (1) the average value of the distance modulus is between $\left(V_{0}-M_{V}\right)_{c l}-0.75-2 \sigma_{V_{0}-M_{V}}$ and $\left(V_{0}-M_{V}\right)_{c l}+2 \sigma_{V_{0}-M_{V}}$ and (2) the difference in the distance moduli is less than $\pm 2.5 \sigma_{V_{0}-M_{V}}$. The factor $-0.75 \mathrm{mag}$ in (1) is added to take into account the effect of equal mass binaries. We adopt the distance moduli of NGC 4609 and Hogg 15 as 10.60 ( \pm 0.08) mag and $12.5( \pm 0.15) \mathrm{mag}$, respectively.

This method only works well when photometry is very accurate on an absolute scale. If the cluster is distant or if there are many field stars with similar reddenings and distances, the usefulness of this method is very limited. In such cases we should first correct for the contribution of field interlopers. To estimate the distance modulus of a star we need to know the reddening. We derive the reddening map of NGC 4609 using the less reddened blue stars and it is shown in Fig. 7. The reddening map of NGC 4609 is very smooth.

We estimate the reddening of faint stars by interpolating the reddening map. We select photometric members of NGC 4609 and Hogg 15 down to $V=17$ mag. The stars in the region observed only on 1997 March 2 (northern edge and eastern edge of Fig. 1) have $U B V$ data only, and therefore the same photometric membership selection criteria cannot be applied. The photometric members of these regions have been selected only from the $\left[V_{0},(B-V)_{0}\right]$. These stars are marked as the smallest circles in Fig. 4 or 9 .
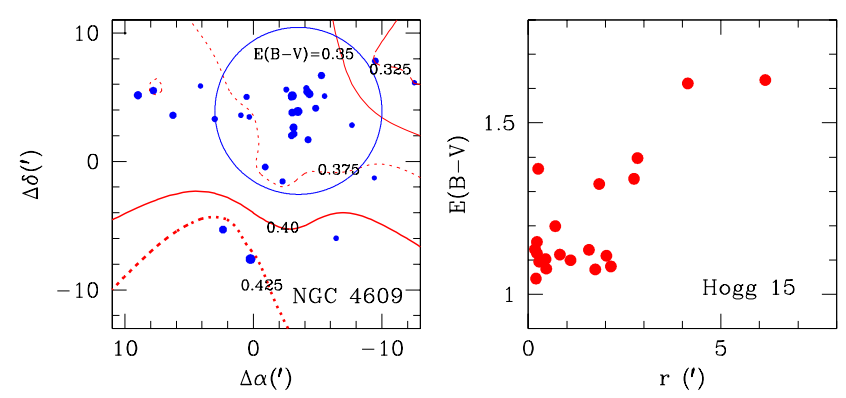

Fig. 7.- Reddening map of NGC 4609 and the variation of $E(B-V)$ within Hogg 15 .

\subsection{2 $N G C 4609$}

Fig. 8 shows the distribution of distance moduli of photometric members in NGC 4609. The thin solid histogram represents the distribution of distance moduli of photometric members within the cluster radius, while the dotted histogram shows the distribution of distance moduli of stars outside the cluster radius which meet the same photometric membership selection criteria. The thick solid histogram represents the distribution of distance moduli subtracted for the contribution of field stars. The dotted histogram in Fig. 8 shows a

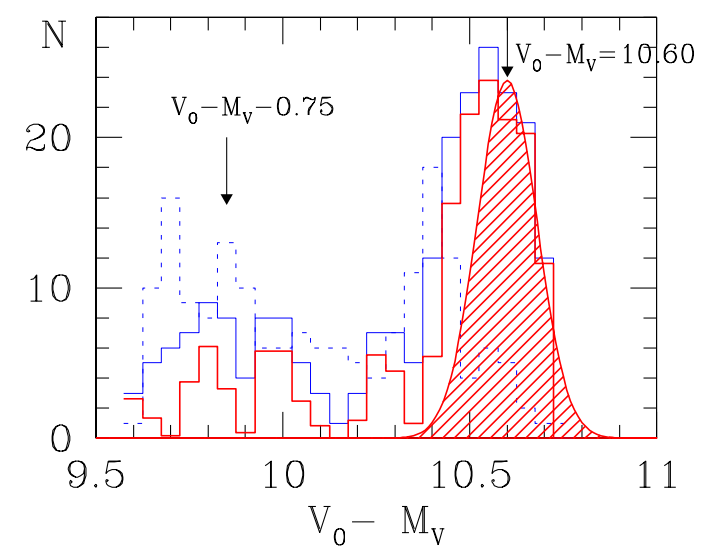

Fig. 8. - The distribution of distance moduli of stars in and around NGC 4609. The thin solid and dotted histograms represent the distribution of distance moduli of photometric members within and outside NGC 4609, respectively. The thick solid histogram shows the distribution of distance moduli subtracted for the contribution of field stars. The hatched area shows a gaussian function fit to the distribution of the right hand portion which approximates the distance modulus distribution of single stars.

slight shift to the smaller distance modulus relative to the thin solid histogram. This fact implies that the field stars having similar photometric characteristics to NGC 4609 are slightly brighter than the stars in NGC 4609 , which relates to the finding of Feinstein \& Marraco (1971) that there is a large spread in the distance moduli of stars in NGC 4609.

The shaded area in Fig. 8 represents a gaussian function fit to the distance moduli distribution to estimate the distance modulus and the binary fraction of NGC 4609. The distance modulus of NGC 4609 from the gaussian function fit is $10.60 \pm 0.08 \mathrm{mag}$ ( $d$ $=1.32 \pm 0.05 \mathrm{kpc}$ ). This value is the same as the distance determined by Feinstein \& Marraco (1971). The best fit gaussian function can account for 47.6 stars amongst a total of 81.3 photometric members. The binary fraction of NGC 4609 is therefore $41.5 \%$ (= $100 \times(81.3-47.6) / 81.3)$. Two factors may affect this fraction. Contamination by field stars may cause the binary fraction to be overestimated because field stars seem to be relatively older than NGC 4609 and are therefore slightly brighter. Another is the effect of binary systems with a large mass ratio. In that case, the light contribution from the faint companion is insignificant and we may underestimate the binary fraction. At this point we cannot estimate which effect affects the results more.

Fig. 9 shows the CMDs of the observed region. The blue members as well as photometric members of each cluster are marked with different symbols. There are several photometric members with red $J-H$ colors. 

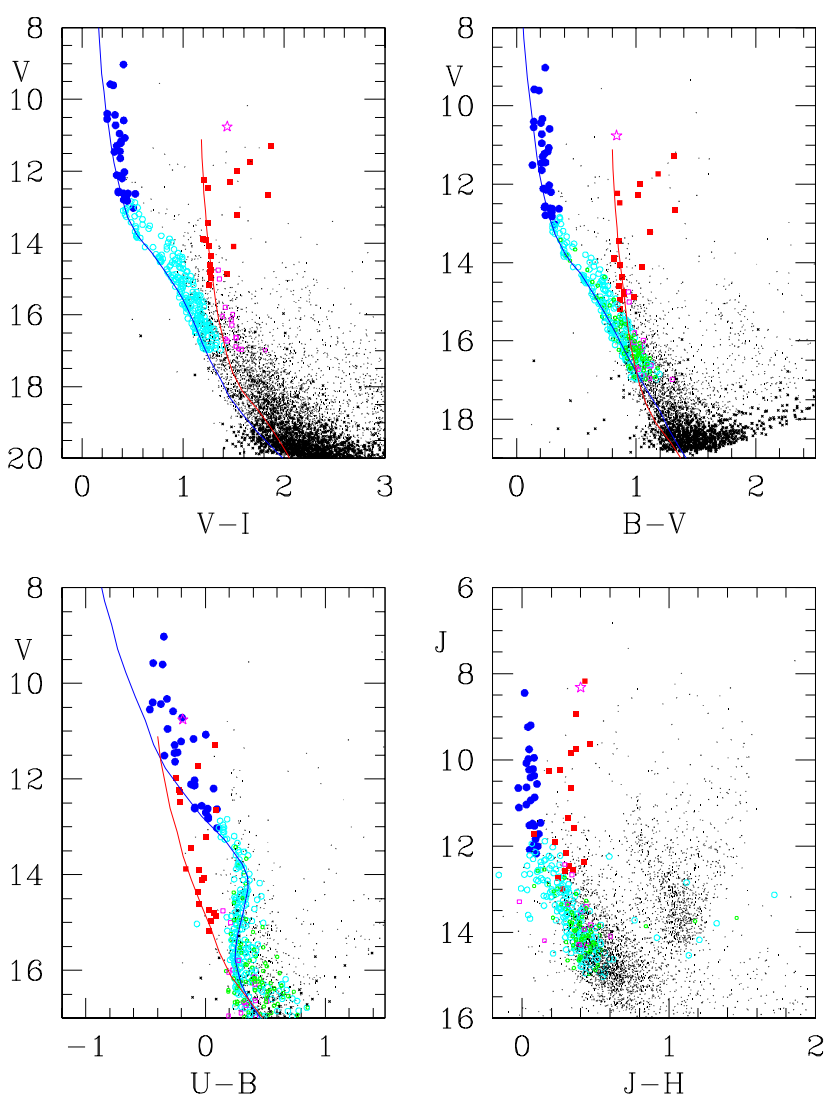

Fig. 9. - Color-magnitude diagrams. Two solid lines represent the ZAMS relations reddened by $E(B-V)=0.37$ and $V_{0}-M_{V}=10.6$, and $E(B-V)=1.13$ and $V_{0}-M_{V}=12.5$, respectively. All the other symbols are the same as in Fig. 4.

Most of these are due to the photometric errors in 2MASS (upper limit in $H$ ). Only two stars (ID 160 $=$ 2MASS J12410653-6304343 and ID $6987=2$ MASS J12440630-6303586) seem to have normal optical colors and red near-IR colors. Their nature should be checked by spectroscopic observations.

\subsubsection{Hogg 15}

The radial variation of reddening in the Hogg 15 region is shown in the right panel of Fig. 7. The amount of reddening near the cluster center is small, but increases abruptly outside $r \approx 2^{\prime}$. A similar variation pattern can be seen in the starburst-type young open cluster NGC 3603 (see Fig. 5 of Sung \& Bessell 2004). Such a variation may be caused by strong winds from young massive stars.

The distance modulus of Hogg 15 is estimated to be $12.50 \pm 0.15 \mathrm{mag}(d=3.2 \pm 0.2 \mathrm{kpc})$ from the $\left(M_{V}\right.$, $B-V)$ and $\left(M_{V}, U-B\right)$ relations for the ZAMS stars and the reddening-corrected magnitude and colors of young blue stars in Hogg 15. This value is somewhat smaller than the distance obtained by Moffat (1974 $d=4.2 \pm 0.4 \mathrm{kpc})$ and van der Hucht $(2001-d=3.80$ kpc). Sagar et al. (2001) estimated the distance to Hogg 15 as $3.0 \pm 0.3 \mathrm{kpc}$, while Piatti \& Clariá (2001) obtained a much smaller value $(d=2.6 \pm 0.08 \mathrm{kpc})$. We have selected photometric members of the Hogg 15 group in the restricted area $\left(\Delta \alpha=-5^{\prime}-10^{\prime}\right.$ and $\left.\Delta \delta=-5^{\prime}--^{\prime}\right)$ where we could find highly reddened early-type stars. Only 13 and 3 photometric members, respectively, have been selected within and outside the cluster radius.

\section{AGE AND MASS FUNCTION}

\section{$4.1 \quad$ NGC 4609}

\subsubsection{Age of $N G C 4609$}

Feinstein \& Marraco (1971) determined the age of NGC 4609 (age $\left.=6 \times 10^{7} \mathrm{yrs}\right)$. In order to determine the age of NGC 4609 using the most recent isochrones we have drawn the observational Hertzsprung-Russell (H-R) diagram of NGC 4609 in Fig. 10. Superimposed are the most recent isochrones from the Padua group (Bertelli et al. 2009) for solar abundances (upper two panels) and for $\mathrm{Z}=0.008$ (lower two panels). Clariá et al. (1989) observed a red giant candidate in NGC 4609 - No 43 of Feinstein \& Marraco (1971) - in $U B V$, DDO, and Washington systems, but they did not arrive at any conclusion regarding the metallicity of NGC 4609. Although the star is outside the cluster boundary, they assumed it to be a member of NGC 4609 from its $E(B-V)$ and spectral type estimated from DDO photometry. As mentioned in Section 3.3.1, the photometric characteristics of members and field stars around NGC 4609 are very similar, and so the reddening $E(B-V)$ by itself cannot be a decisive membership criterion.

As mentioned in Cheon et al. (2010), the ZAMS of the solar abundance models of Padua group is brighter than the empirical one by about 0.3 mag. In spite of the difference in abundances and/or ZAMS relations, the age of NGC 4609 is well fitted to the isochrone of age $5 \times 10^{7}$ yrs. As there is only a small difference in the age from both diagrams we therefore adopt the age of NGC 4609 as log age $=7.7 \pm 0.1$.

\subsubsection{Luminosity and Mass Function of NGC 4609}

The slope between the absolute magnitude and mass, i.e. $\frac{d M_{V}}{d \log m}$, is relatively large for intermediateor low-mass stars. It is easy to estimate the mass of a star in intermediate-age open clusters reliably from its absolute magnitude. We first calculate the luminosity function (LF) of the photometric members of NGC 4609 after subtracting the contribution of field stars. The LF of NGC 4609 is calculated with the bin size $\left(\Delta M_{V}\right)$ of 1 mag. And we also calculate the LF with the same bin size but shifted by 0.5 mag to minimize 

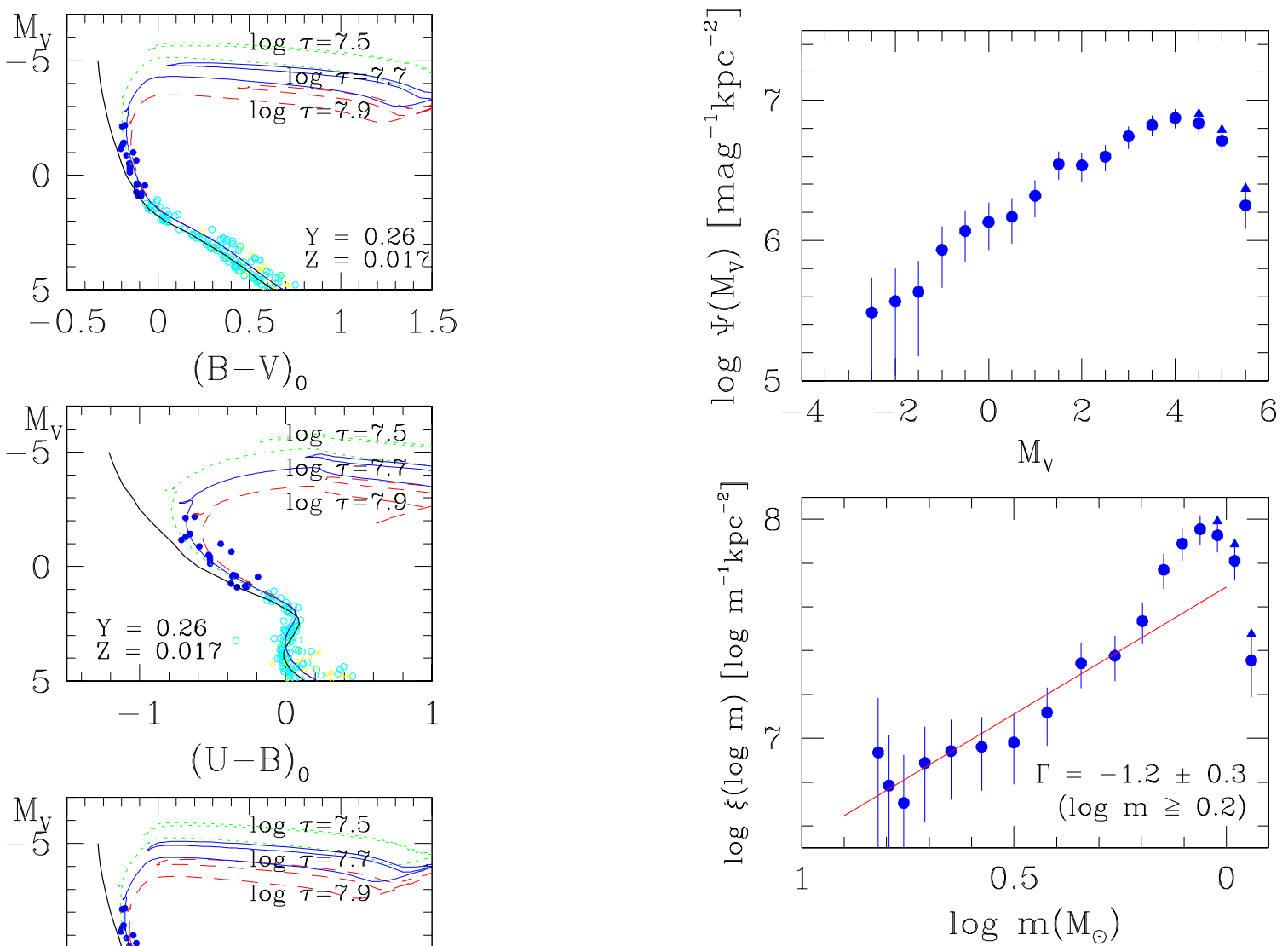

Fig. 11. - The luminosity and mass function of NGC 4609 .

the effect of binning. The LF of NGC 4609 is presented in the upper panel of Fig. 11.

The mass function (MF) of NGC 4609 can be derived from the relation $\xi(\log m) d \log m \equiv \Psi\left(M_{V}\right) d M_{V}$ and the adopted mass-luminosity relation. In calculating the MF of NGC 4609, we use the mass-luminosity relation of the isochrone of $\log a g e=7.7$ and $\mathrm{Z}=$ 0.008 (Bertelli et al. 2009). The MF obtained here is shown in the lower panel of Fig. 11. The slope of the $\mathrm{MF}$ for $\log m \geq 0.2$ is $-1.2 \pm 0.3$. For low-mass stars $(\log m<0.2)$ some enhancement is readily seen which may be attributable to contamination from field stars. As already mentioned in Section 3.3.1, photometric membership criteria do not work well if the photometric characteristics of cluster stars and field stars are similar. For brighter stars photometry is relatively accurate, and so the photometric membership criteria work well. But for fainter stars the error in photometry increases rapidly, and therefore we can expect the inevitable contamination by field stars. $\left(r<6 .^{\prime} 5\right)$. The superimposed isochrones of $\log$ age $=7.5$, 7.7, and 7.9 from Bertelli et al. (2009) are for solar abundances (upper two panels) and for $\mathrm{Z}=0.008$ (lower two panels). The thick solid line represents the empirical ZAMS relation. All other symbols are the same as in Fig. 4. 


\section{$4.2 \quad$ Hogg 15}

\subsubsection{The Age of Hogg 15}
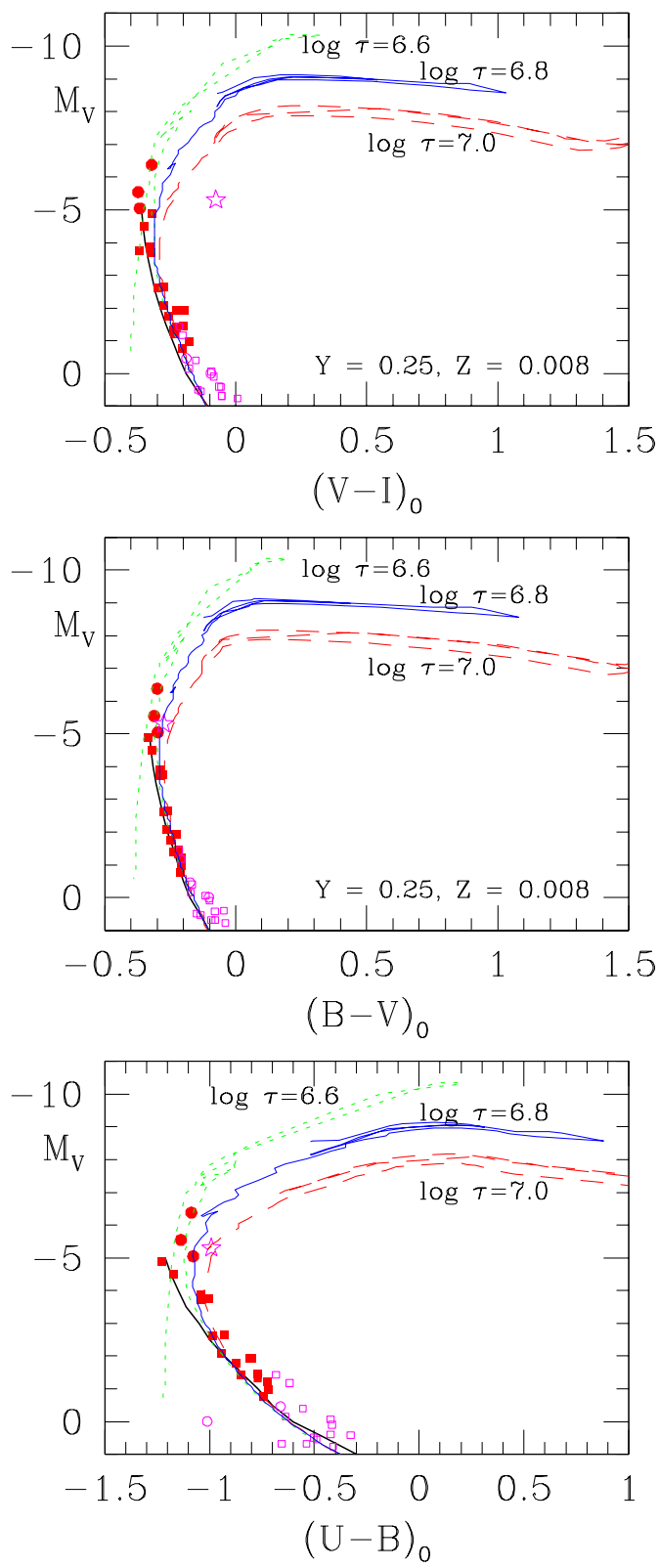

Fig. 12. - The observational H-R diagrams of Hogg 15 . The thick solid line represents the empirical ZAMS relation, while the thin dotted, solid, and dashed lines are the isochrones of $\log a g e=6.6,6.8$, and 7.0, respectively for $\mathrm{Z}=0.008$ from Bertelli et al. (1994). Squares and circles denote the member stars within and outside of Hogg 15, respectively. Filled and open symbols represent the young blue stars and less bright photometric members, respectively. The star symbol represents the position of WR 47 .
Moffat (1974) estimated the age of Hogg 15 as $\lesssim 8 \times 10^{6}$ yrs probably from the earliest photometric spectral type $(b 0)$ of stars in Hogg 15. Later Sagar et al. (2001) determined the age as $6 \pm 2 \mathrm{Myr}$, but Piatti \& Clariá (2001) derived a much older age $(\sim 300$ Myr). We constructed the observational H-R diagram of Hogg 15 in Fig. 12. As Bertelli et al. (2009) do not provide isochrones for $\log a g e<7.0$, we superimpose the isochrones of a younger age from Bertelli et al. (1994). For OB stars, metallicity hardly affects the optical colors; we use the isochrones for $\mathrm{Z}=0.008$.

Filled and open symbols in Fig. 12 represent stars within and outside the radius of Hogg 15 . The stars in Hogg 15 are very close to the empirical ZAMS, and younger than the youngest isochrone $(\log$ age $=6.6)$. The young stars outside the radius of Hogg 15 are on the isochrone of $\log a g e=6.6$. Although WR 47 (star symbol in Fig. 12) seems to be older than the others, its position in Fig. 12 cannot indicate the age of Hogg 15 because its colors may be affected by the emission lines of WN6 stars. The age of Hogg 15 is younger than or similar to 4 Myr $(\log$ age $\lesssim 6.6)$.

\subsubsection{The Initial Mass Function of Hogg 15}

The mass of individual members in Hogg 15 is estimated using the Padua isochrone of log age $=6.6$. The mass of WR 47 (WN6 + O5:V) is from Lamontagne et al. (1996). Although the observed mass of WN6 stars is $51 \mathrm{M}_{\odot}$, its initial mass is much larger than its companion $\left(\mathrm{O} 5: \mathrm{V}-60 \mathrm{M}_{\odot}\right)$. We tentatively assume, therefore, that the mass of the WN6 star is $80 \mathrm{M}_{\odot}$ (see Section 5.1 for more discussion).

The initial mass function (IMF) of Hogg 15 is derived and presented in Fig. 13. For Hogg 15 we do not attempt to subtract the contribution of field stars because the photometric members are blue stars and

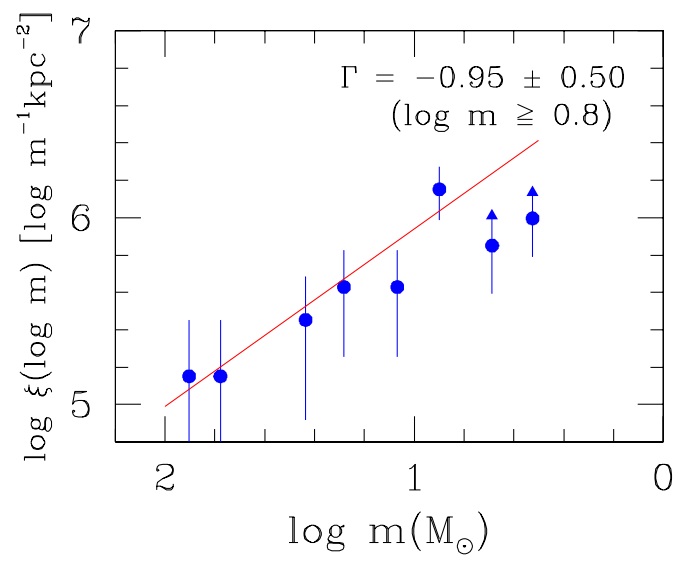

Fig. 13. - The initial mass function of Hogg 15 . 
such blue stars are not found in the field. ${ }^{\ddagger}$ The slope of the IMF is $-0.95 \pm 0.50$ for $\log m \geq 0.8$, which is somewhat shallower than normal. The large error is caused by the small number of member stars.

\section{DISCUSSION}

\subsection{Age and Initial Mass of the WN6 Stars in Hogg 15}

To estimate the initial mass of the WN6 star in WR 47, we have drawn the upper part of the H-R diagram in Fig. 14. The theoretical stellar evolution models with rotation constructed by the Geneva group (Meynet \& Maeder 2003) are superimposed. The absolute visual magnitude of WR 47 is $-5.30 \mathrm{mag}$ if we apply the $E(B-V)$ derived from Fig. 4 by assuming the broad-band color of the system is dominated by light from the normal companion $\mathrm{O} 5: \mathrm{V}$ star. We estimate the absolute bolometric magnitude of $\mathrm{O} 5: \mathrm{V}$ in two ways - (1) both O5:V and WN6 equally contribute to the total light, and (2) the light from O5:V star dominates in the WR 47 system. The open circle in Fig. 14 is for case (1), and the filled circle is for case (2). The error bar is for a 1 subclass error in the spectral type of the $\mathrm{O} 5: \mathrm{V}$ star, i.e. $\mathrm{O} 4 \mathrm{~V}-\mathrm{O} 6 \mathrm{~V}$. The mass and age of the O5:V star are,

case (1): $\left(\sim 32 \mathrm{M}_{\odot}, \sim 1.7 \mathrm{Myr}\right)-\left(\sim 40 \mathrm{M}_{\odot}, \sim \mathrm{ZAMS}\right)$ case (2): $\left(\sim 40 \mathrm{M}_{\odot}, \sim 2.9 \mathrm{Myr}\right)-\left(\sim 52 \mathrm{M}_{\odot}, \sim 1.3 \mathrm{Myr}\right)$

The most massive star in the Geneva models (120 $\mathrm{M}_{\odot}$, total lifetime : $3.55 \mathrm{Myr}$ ) becomes an early phase WNL at about $2.15 \mathrm{Myr}\left(\sim 86 \mathrm{M}_{\odot}\right)$, a WNE at about $3.19 \mathrm{Myr}\left(\sim 23.5 \mathrm{M}_{\odot}\right)$, and a $\mathrm{WC}$ at about 3.26 $\mathrm{Myr}(\sim$ $20.5 \mathrm{M}_{\odot}$ ). And a $85 \mathrm{M}_{\odot}$ star (total lifetime : $4.09 \mathrm{Myr}$ ) becomes WNL, WNE, WN/WC, and WC phase at 2.68 $\operatorname{Myr}\left(\sim 64.6 \mathrm{M}_{\odot}\right), 3.75 \mathrm{Myr}\left(\sim 20.8 \mathrm{M}_{\odot}\right), 3.88 \mathrm{Myr}(\sim$ $\left.17.3 \mathrm{M}_{\odot}\right)$, and $3.885 \mathrm{Myr}\left(\sim 17.1 \mathrm{M}_{\odot}\right)$, respectively. The observational mass ratio $\left(q_{W R}\right)$ is 0.85 .

The maximum age of case (1) is $1.7 \mathrm{Myr}$ which is untenable because even a $120 \mathrm{M}_{\odot}$ star cannot evolve into the WNL phase in this time. There are three possibilities for case (2). The most massive case $\left(\sim 52 \mathrm{M}_{\odot}\right.$ for the companion) is also unacceptable because of the age. At the age of $2.2 \mathrm{Myr}$ for O5 star, a $120 \mathrm{M}_{\odot}$ star would have just evolved into the WNL phase, and have a mass of about $83.7 \mathrm{M}_{\odot}$, which is much larger than the mass of the WN6 $\operatorname{star}\left(q_{W R} \cdot \mathrm{M}_{O 5: V} \approx 38 \mathrm{M}_{\odot}\right)$.

The only remaining case is the case of $\left(\mathrm{M}_{O 5: V} \sim 40\right.$ $\mathrm{M}_{\odot}$, age $\left.\sim 2.9 \mathrm{Myr}\right)$. The mass of the WN6 is $q_{W R}$. $\mathrm{M}_{O 5: V} \approx 34 \mathrm{M}_{\odot}$, and the evolutionary mass of $\mathrm{m}_{\text {init }}=$ $120 \mathrm{M}_{\odot}$ at $2.9 \mathrm{Myr}$ is $36.2 \mathrm{M}_{\odot}$. These two values are very similar. In addition, the maximum mass loss from the current mass loss rate (Moffat et al. 1990) is about $87 \mathrm{M}_{\odot}\left(=\dot{M} \cdot \tau_{\text {age }}=3 \times 10^{-5} \mathrm{M}_{\odot} y r^{-1} \cdot 2.9 \times 10^{6} y r\right)$. This value is consistent with the mass obtained above $\left(\mathrm{M}_{W N 6}>33 \mathrm{M}_{\odot}=120 \mathrm{M}_{\odot}-87 \mathrm{M}_{\odot}\right)$. We obtain a

$\ddagger$ The young blue stars outside the radius of Hogg 15 may be members of an unclassified association around Hogg 15.

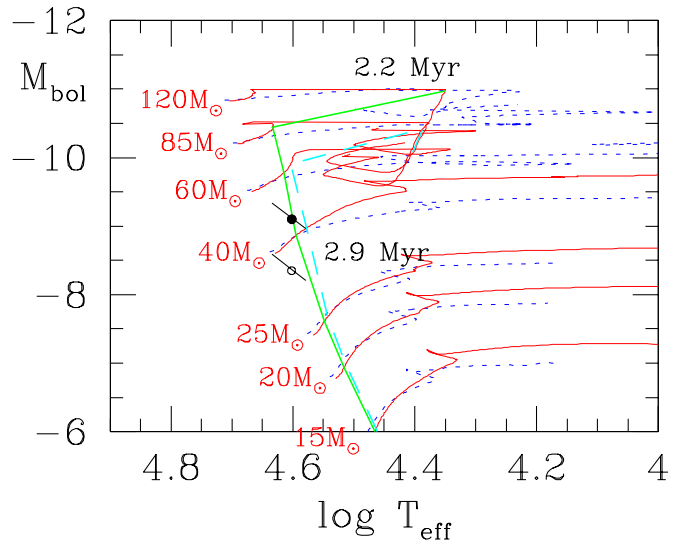

Fig. 14. - The H-R diagram of the O5:V star in WR 47. Thin solid and dotted lines with mass to the left are stellar evolution models from Meynet \& Maeder (2003) with and without stellar rotation, respectively. Thick solid and dashed lines represent the isochrones from evolution models with stellar rotation $\left(v_{\text {rot }}=300 \mathrm{~km} \mathrm{~s}^{-1}\right)$ of age 2.2 and 2.9 Myr, respectively. Open and filled circles are the position of the O5:V star for two cases. See text for details.

much smaller mass for the WR 47 system $\left(\mathrm{M}_{W R} 47=\right.$ $74 \mathrm{M}_{\odot}$ from this) compared to the $111 \mathrm{M}_{\odot}$ in Lamontagne et al. (1996). Niemela et al. (1980) derived mass ratios and masses for several cases. But for all cases the total mass of the system was larger than 82 $\mathrm{M}_{\odot}$.

In this calculation we have implicitly assumed that the mass ratio obtained from observations is correct, that the mass of individual components may be incorrect, that the WR system is coeval, and that the stellar evolution models are correct. In addition, we assumed no mass transfer between components. The above result may be meaningless if even one of the above assumptions are invalid. Another problem that needs to be solved is the bolometric correction (BC) scale of WR stars. The $\mathrm{M}_{b o l}$ of $\mathrm{m}_{\text {init }}=120 \mathrm{M}_{\odot}$ at $2.9 \mathrm{Myr}$ is about -10.1 mag. The absolute visual magnitude of the WN6 star will be -4.7 mag if we apply BC $=-5.4 \mathrm{mag}$ (Nugis \& Lamers 2000). In this case the light from the WN6 star dominates that from the $\mathrm{O} 5: \mathrm{V}$ star.

The predictions from stellar evolution models and observations are inconsistent for the WR 47 system. As the absolute parameters for each star in the system are very important in the observational tests of the formation and evolution of massive stars, more observational studies should be made to clarify the evolutionary status of WR 47.

\section{SUMMARY}

$U B V I$ CCD photometry data has been obtained for the intermediate-age open cluster NGC 4609 and the 
young open cluster Hogg 15. The results obtained may be summarized as follows.

1. A nearly normal reddening law $\left(R_{V}=3.2\right)$ is determined for the observed region from the color excess ratios of highly reddened early type stars in the observed region.

2. The distance and median value of interstellar reddening of NGC 4609 are $10.60 \pm 0.08 \mathrm{mag}(d=$ $1.32 \pm 0.05 \mathrm{kpc}$ ) and $0.37 \pm 0.03 \mathrm{mag}$, respectively. The corresponding parameters of Hogg 15 are $12.5 \pm$ $0.15 \mathrm{mag}(d=3.2 \pm 0.2 \mathrm{kpc})$ and $1.13 \pm 0.11 \mathrm{mag}$.

3. The radius of NGC 4609 is estimated to be $r=$ $6 . ' 5$ from the surface density variation of bright $B$ type stars in the observed region. That of Hogg 15 is also determined to be $r=3 .^{\prime} 5$. Hogg 15 is found to be a cluster with a core and a halo surrounding the core.

4. The WR star HDE 311884 is in the halo of Hogg 15. Its reddening, brightness and age of Hogg 15 strongly imply that the star is a member of Hogg 15.

5. Using the photometric membership selection criteria, we select cluster members down to $V=17$ mag.

6. The age and mass function of NGC 4609 and Hogg 15 were derived. The age of NGC 4609 is $\log$ age $=7.7 \pm 0.1$, while that of Hogg 15 is log age $\lesssim$ 6.6. The slope of the mass function of NGC 4609 is nearly normal, but in the low-mass regime the mass function seems to be affected by field interlopers. On the other hand, the IMF slope of Hogg 15 is slightly shallow $(\Gamma=-0.95 \pm 0.5)$.

7. We have also discussed the mass and evolutionary status of the double-line binary system WR 47 (WN6 $+\mathrm{O} 5: \mathrm{V})$.

\section{ACKNOWLEDGMENTS}

H. S. acknowledges the support of the National Research Foundation of Korea (NRF) to the Astrophysical Research Center for the Structure and Evolution of the Cosmos (ARCSEC ${ }^{\prime \prime}$ ) at Sejong University (NRF No. 2009-0062865).

\section{REFERENCES}

Bertelli, G., Bressan, A., Chiosi, C., Fagotto, F., \& Nasi, E. 1994, Theoretical Isochrones from Models with New Radiative Opacities, A\&AS, 106, 275

Bertelli, G., Nasi, E., Girardi, L., \& Marigo, P. 2009, Scaled Solar Tracks and Isochrones in a Large Region of the Z-Y Plane. II. from 2.5 to $20 \mathrm{M}_{\odot}$ Stars, A\&A, 508, 355

Cheon, S., Sung, H., \& Bessell, M. S. 2010, No Open Cluster in the Ruprecht 93 Region, JKAS, 43, 115

Clariá, J. J., Lapasset, E., \& Minniti, D. 1989, Photometric Metal Abundances of High-Luminosity Red Stars in Young and Intermediate-Age Open Clusters, A\&AS, 78, 363
Feinstein, A., \& Marraco, H. G. 1971, The Open Cluster NGC 4609 behind the Coalsack, PASP, 83, 218

Guetter, H. H., \& Vrba, F. J. 1989, Reddening and Polarimetric Studies toward IC 1805, AJ, 98, 611

Hou, L. G., Han, J. L., \& Shi, W. B. 2009, The Spiral Structure of Our Milky Way Galaxy, A\&A, 499, 473

Van der Hucht, K. A. 2001, The VII-th Catalogue of Galactic Wolf-Rayet Stars, New Astronomy Reviews, 45, 135

Humphreys, R. M. 1978, Studies of Luminous Stars in Nearby Galaxies. I. Supergiants and O stars in the Milky Way, ApJS, 38, 309

Lamontagne, R. \& Moffat, A. F. J. 1987, Precision Photometric Monitoring of Southern Variable WolfRayet Stars with a Comment on the Overall Continuum Variability of WR stars, AJ, 94, 1008

Lamontagne, R., Moffat, A. F. J., Drissen, L., Robert, C., \& Mathews, J. M. 1996, Photometric Determination of Orbital Inclinations and Mass Loss Rates for Wolf-Rayet Stars in WR+O Binaries, AJ, 112, 2227

Landolt, A. U. 1992, UBVRI Photometric Standard Stars in the Magnitude Range $11.5<V<16.0$ around the Celestial Equator, AJ, 104, 340

Lim, B., Sung, H., Bessell, M. S., Karimov, R., \& Ibrahimov, M. 2009, CCD Photometry of Standard Stars at Maidanak Astronomical Observatory in Uzbekistan: Transformations and Comparisons, JKAS, 42, 161

Lodén, L. O. 1979, Continued Studies of Loose Clusterings in the Southern Milky Way, A\&AS, 38, 355

Massey, P., DeGioia-Eastwood, K., \& Waterhouse, E. 2001, The Progenitor Masses of Wolf-Rayet Stars and Luminous Blue Variables Determined from Cluster Turnoffs. II. Results from 12 Galactic Clusters and OB Associations, AJ, 121, 1050

Menzies, J. W., Marang, F., Laing, J. D., Coulson, I. M., \& Engelbrecht, C. A. 1991, $U B V(R I)_{C}$ Photometry of Equatorial Standard Stars. A Direct Comparison between the Northern and Southern Systems, MNRAS, 248, 642

Meynet, G., \& Maeder, A. 2003, Stellar Evolution with Rotation X. Wolf-Rayet Star Populations at Solar Metallicity, A\&A, 404, 975

Moffat, A. F. J. 1974, The Wolf-Rayet Star HDE 311884 in the Open Cluster Hogg 15, A\&A, 34, 29

Moffat, A. F. J. et al. 1990, Photometric and Polarimetric Variability and Mass-Loss Rate of the Massive Binary Wolf-Rayet Star HDE 311884 (WN6 + O5:V), ApJ, 350, 767

Muzzio, J. C., Feinstein, A., \& Orsatti, A. M. 1976 , $U B V$ and $\mathrm{H} \beta$ Photometry of Faint Early Type Stars in Crux and Centaurus, PASP, 88, 526 
Niemela, V. S., Conti, P. S., \& Massey, P. 1980, HDE 311884: A Massive Wolf-Rayet Binary, ApJ, 241, 1050

Nugis, T., \& Lamers, H. J. G. L. 2000, Mass-Loss Rates of Wolf-Rayet Stars as a Function of Stellar Parameters, A\&A, 360, 227

Orsatti, A. M., Vega, E., \& Marraco, H. G. 1998, Polarimetry of the Highly Reddened Open Clusters Hogg 15 and Lyngå14, AJ, 116, 266

Piatti, A. E., \& Clariá, J. J. 2001, On the Stellar Content of the Open Clusters Melotte 105, Hogg 15, Pismis 21 and Ruprecht 140, A\&A, 370, 931

Sagar, R., Munari, U., \& de Boer, K. S. 2001, A Multicolour CCD Photometric and Mass Function Study of the Distant Southern Open Clusters NGC 3105, NGC 3603, Melotte 105, Hogg 15, NGC 4815, Pismis 20 and NGC 6253, MNRAS, 327, 23

Smith, M. A., \& Balona, L. 2006, The Remarkable Be Star HD 110432 (BZ Crucis), ApJ, 640, 491

Sung, H., \& Bessell, M. S. 1999, UBVI CCD Photometry of M35 (NGC 2168), MNRAS, 306, 361

Sung, H., \& Bessell, M. S. 2004, The Initial Mass Function and Stellar Content of NGC 3603, AJ, 127, 1014

Sung, H., Bessell, M. S., Chun, M.-Y., Karimov, R., \& Ibrahimov, M. 2008, The Initial Mass Function and Young Brown Dwarf Candidates in NGC 2264. III. Photometric Data, AJ, 135, 441

Sung, H., Bessell, M. S., Lee, B.-W. \& Lee, S.-G. 2002 , The Open Cluster NGC 2516 - 1. Optical Photometry, AJ, 123, 290

Sung, H., Bessell, M. S., Lee, H.-W., Kang, Y. H., \& Lee, S.-W. 1999, UBVI CCD Photometry of M11. II. New Photometry and Surface Density Profiles, MNRAS, 310, 982

Sung, H., Bessell, M. S., \& Lee, S.-W. 1998, UBVRI and $\mathrm{H} \alpha$ Photometry of the Young Open Cluster NGC 6231, AJ, 115, 734 